\title{
General Relation Connecting the Fundamental Fields
}

\author{
Mukul Chandra Das ${ }^{1 *}$, Rampada Misra ${ }^{2}$ \\ ${ }^{1}$ Singhania University, Jhunjhunu, India \\ ${ }^{2}$ Department of Electronics, Vidyasagar University, Midnapore, West Bengal, India \\ Email: *mukuldas.100@gmail.com
}

Received June 3, 2012; revised July 18, 2012; accepted July 25, 2012

\begin{abstract}
There are four fundamental forces: gravitational force, electromagnetic force, strong force and weak force, in the well known physics. The unified field theory considers the constructive relations among these forces or fields. In the present work the fundamental relations have been studied and trial has been made to derive more significant relations among the known fields. This gives out a generalized unification.
\end{abstract}

Keywords: Fundamental Force; Unified Field; Generalized Relation

\section{Introduction}

According to Newton's law, two bodies of mass $m_{1}$ and $m_{2}$ attract one another with gravitational force whose magnitude is $F_{\text {grav }}=\frac{G m_{1} m_{2}}{r^{2}}$. But Einstein's general relativity does not consider gravity as a force rather it is a space-time curvature. As in [1] Newtonian field equation is $\nabla^{2} \Phi=4 \pi G \mu$, but in general relativity the Einstein equation is $R_{\alpha \beta}-\frac{1}{2} g_{\alpha \beta} R=8 \pi G T_{\alpha \beta}$. On the other hand Maxwell equations [2] are the field equations of electromagnetism that relate the electromagnetic field to its source-charge and current. But Einstein's equation relates the space-time curvature to its source- the mass-energy of matter. The well known unified electromagnetic field Equations [2] are $\boldsymbol{E}=\gamma\left(\boldsymbol{E}^{\prime}+\boldsymbol{v} \times \boldsymbol{B}^{\prime}\right)$ and

$\boldsymbol{B}=\gamma\left(\boldsymbol{B}^{\prime}+\frac{\boldsymbol{v} \times \boldsymbol{E}^{\prime}}{c^{2}}\right)$. These imply that one observer's electric field is another's magnetic field and that depends on the relativity. In 1935, H. Yukawa proposed a theory on generation of strong force [3] which deals with particle physics. This theory implies a relation between electromagnetic field and strong field. After a long year of this contribution, the weak force and the electromagnetic force were unified in a theory presented independently by A. Salam, Weinberg and Glashow [4-6]. Afterwards a lot of papers, regarding unified field theory, have been published. However, in $[7,8]$, trial have been made to deduce relations among the known fields (i.e. gravitational field, electromagnetic field, strong field) following a constructive method, which may satisfy the dream of

"Corresponding author.
Einstein's fields unification. The present work is the modified formulation of unified field equations as discussed in $[7,8]$.

\section{Modified Relation among the Fields}

The well known relations between electric field and magnetic field are

$$
\begin{aligned}
\boldsymbol{E} & =\boldsymbol{v} \times \boldsymbol{B} \\
\boldsymbol{B} & =\frac{\boldsymbol{v} \times \boldsymbol{E}}{c^{2}}
\end{aligned}
$$

From (1) and (2) we shall have the matrix form of these field transformation as

$$
\left(\begin{array}{l}
E_{x} \\
E_{y} \\
E_{z}
\end{array}\right)=k_{1} v_{i j}\left(\begin{array}{l}
B_{x}^{\prime} \\
B_{y}^{\prime} \\
B_{z}^{\prime}
\end{array}\right)
$$

where $v_{i j}=\left(\begin{array}{ccc}v_{x x} & v_{x y} & v_{x z} \\ v_{y x} & v_{y y} & v_{y z} \\ v_{z x} & v_{z y} & v_{z z}\end{array}\right), k_{1}$ and $k_{2}$ are two constants. Again, we would obtain from relativistic electrodynamics [2] the relations

$$
\begin{aligned}
& \boldsymbol{E}=\gamma \boldsymbol{v} \times \boldsymbol{B} \\
& \boldsymbol{B}=\gamma \frac{\boldsymbol{v} \times \boldsymbol{E}}{c^{2}}
\end{aligned}
$$

where, $\gamma \boldsymbol{v}=\boldsymbol{V}$ is the proper velocity. So, using (3) and 
(4) we get from (5) and (6)

$$
\begin{aligned}
& \left(\begin{array}{l}
E_{x} \\
E_{y} \\
E_{z} \\
E_{t}
\end{array}\right)=\chi_{1} V_{i j}\left(\begin{array}{c}
B_{x}^{\prime} \\
B_{y}^{\prime} \\
B_{z}^{\prime} \\
B_{t}^{\prime}
\end{array}\right) \\
& \left(\begin{array}{l}
B_{x} \\
B_{y} \\
B_{z} \\
B_{t}
\end{array}\right)=\chi_{2} V_{i j}\left(\begin{array}{c}
E_{x}^{\prime} \\
E_{y}^{\prime} \\
E_{z}^{\prime} \\
E_{t}^{\prime}
\end{array}\right)
\end{aligned}
$$

$\chi_{1}$ and $\chi_{2}$ are also two constants.

where, $V_{i j}=\left(\begin{array}{cccc}V_{x x} & V_{x y} & V_{x z} & V_{x t} \\ V_{y x} & V_{y y} & V_{y z} & V_{y t} \\ V_{z x} & V_{z y} & V_{z z} & V_{z t} \\ V_{t x} & V_{t y} & V_{t z} & V_{t t}\end{array}\right)$

But, $\boldsymbol{E}$ and $\boldsymbol{B}$ are not separate. These are included in a field which is called electromagnetic field. According to $[9,10]$ electromagnetic field function $\boldsymbol{\psi}=\boldsymbol{E}+i \boldsymbol{B}$. So, from (7) and (8) we get a generalized relation

$$
\psi_{\alpha}(\boldsymbol{E}, \boldsymbol{B})=\Phi V_{i j} \psi_{\alpha}^{\prime}\left(E^{\prime}, B^{\prime}\right)
$$

$$
\text { where, } \psi_{\alpha}(\boldsymbol{E}, \boldsymbol{B})=\left(\begin{array}{c}
\psi_{x} \\
\psi_{y} \\
\psi_{z} \\
\psi_{t}
\end{array}\right), \psi_{\alpha}^{\prime}(\boldsymbol{E}, \boldsymbol{B})=\left(\begin{array}{c}
\psi_{x}^{\prime} \\
\psi_{y}^{\prime} \\
\psi_{z}^{\prime} \\
\psi_{t}^{\prime}
\end{array}\right)
$$

This means that $B^{\prime}$ and $E^{\prime}$ in $\psi_{\alpha}^{\prime}$ transfer to $E$ and $B$ respectively in $\psi_{\alpha}$. In [7] it reveals that through two simultaneous superimposed motions gravitational field transfers to electromagnetic field and the relation is

$$
\psi_{\alpha}=\Upsilon w_{i j} G_{\alpha}^{\prime}
$$

where $G_{\alpha}^{\prime}=\left(\begin{array}{l}G_{x}^{\prime} \\ G_{y}^{\prime} \\ G_{z}^{\prime} \\ G_{t}^{\prime}\end{array}\right), \quad w_{i j}=\left(\begin{array}{llll}w_{x x} & w_{x y} & w_{x z} & w_{x t} \\ w_{y x} & w_{y y} & w_{y z} & w_{y t} \\ w_{z x} & w_{z y} & w_{z z} & w_{z t} \\ w_{t x} & w_{t y} & w_{t z} & w_{t t}\end{array}\right)$, and $\boldsymbol{w}=\boldsymbol{a}+\frac{i}{c} \boldsymbol{b}$ as in [7]. Again in [8] relation between strong field and electromagnetic field is given by

$$
G_{\alpha}=\mathrm{K} w_{i j} \psi_{\alpha}^{\prime}
$$

This leads to a relation between strong gravitational field (strong field) and weak gravitational field $\left(\boldsymbol{G}_{\alpha}^{\prime}\right)$ which is

$$
G_{\alpha}=\Gamma w_{i j} w_{i j}^{\prime} G^{\prime}
$$

Equations (7), (8), (10) and (11) are analogous. So, following (5) and (6) we can write the relations in vecto- rial form as

$$
\begin{aligned}
& \psi_{\alpha}(\boldsymbol{E}, \boldsymbol{B},)=\Phi_{1} \boldsymbol{w} \times \boldsymbol{G}_{\alpha}^{\prime} \\
& \boldsymbol{G}_{\alpha}=\Phi_{2} \boldsymbol{w} \times \psi_{\alpha}^{\prime}\left(E^{\prime}, B^{\prime},\right)
\end{aligned}
$$

where, $\boldsymbol{G}_{\alpha}^{\prime}$ in (13) represents weak gravitational field and $\boldsymbol{G}_{\alpha}$ in (14) represents strong gravitational field or strong field. $\boldsymbol{w}$ is the composed velocity as in [7] as well as four-velocity. In (13) and (14) $\Phi_{1}$ and $\Phi_{2}$ are two constants.

Again from (12), (13) and (14) we can consider the vector relation between strong field and weak gravitational field which would give

$$
\boldsymbol{G}_{\alpha}=\Phi_{3} \boldsymbol{w} \times\left(\boldsymbol{w}^{\prime} \times \boldsymbol{G}_{\alpha}^{\prime}\right)
$$

where, $\Phi_{3}$ is a constant like $\Phi_{1}$ and $\Phi_{2}$

\section{Conclusion}

In this work a constructive vector relation among the fields has been deduced. Equations (13)-(15) represent such relations which can clear the concepts of fields transformations. These also imply that field transformations are associated with relativistic phenomenon in different frames.

\section{Acknowledgements}

Author thanks the authorities and staff of Satmile High School, Satmile-721452, West Bengal, India for their continuous encouragements.

\section{REFERENCES}

[1] J. B. Hartle, “Gravity. An Introduction to Einstein's General Relativity," 3rd Edition, Pearson Education Inc., India, 2009, p. 510.

[2] D. J. Griffiths, "Introduction to Electrodynamics," 7th Edition, Pearson Education Inc., India, 2009, pp. 549-550.

[3] S. N. Ghoshal, "Nuclear Physics," S. Chand \& Company Limited, New Delhi, 2006, pp. 365-430, 881-889.

[4] L. Glashow, "Towards a Unified Theory: Threads in a Tapestry," Reviews of Modern Physics, Vol. 52, No. 3, 1980, pp. 539-543. doi:10.1103/RevModPhys.52.539

[5] A. Salam, "Gauge Unification of Fundamental Force," Reviews of Modern Physics, Vol. 52, No. 3, 1980, pp. 525-538. doi:10.1103/RevModPhys.52.525

[6] S. Weinberg, "Conceptual Foundations of the Unified Theory of Weak and Electromagnetic Interaction," Reviews of Modern Physics, Vol. 52, No. 3, 1980, pp. 515523. doi:10.1103/RevModPhys.52.515

[7] M. C. Das and R. Misra, "Fundamental Way of Charge Formation and Relation between Electromagnetic Field and Gravitational Field," International Journal of Astronomy and Astrophysics, Vol. 2, No. 2, 2012, pp. $97-$ 100. 
[8] M. C. Das and R. Misra, "A Short Note on Alternative Strong Force," International Journal of Astronomy and Astrophysics, Vol. 2, No. 2, 2012, pp. 74-75.

[9] I. I. Popescu, P. Sterian and M. Dobre, "The Photon Wave Function and the Fresnel Formulas," Romanian
Reports in Physics, Vol. 62, No. 2, 2010, pp. 360-368.

[10] R. E. Nistor, "Quantum Aspects of Photon Propagation in Transparent Infinite Homogeneous Media," Romanian Reports in Physics, Vol. 60, No. 3, 2008, pp. 471-491. 